\title{
ESTUDO DO AVANÇO DE INCLUSÕES NÃO-METÁLICAS ATRAVÉS DO TRATAMENTO COM CÁLCIO NO AÇO SAE $1141^{1}$
}

\begin{abstract}
Bruna lost Camacho Wagner Viana Bielefeldt ${ }^{3}$ Antônio Cezar Faria Vilela ${ }^{4}$

\section{Resumo}

Inclusões não metálicas são impurezas presentes, em menor ou maior grau, nos aços, conforme o tamanho, a morfologia e a composição química das mesmas. Se não moderadas, tal situação corrompe as propriedades mecânicas e metalúrgicas dos aços. A modificação de inclusões com ligas à base de cálcio é uma ferramenta fortemente usada para a produção de aços mais limpos. Tal tratamento é fortemente influenciado pela temperatura e composição química do aço. O presente trabalho utilizará como metodologia: i) obtenção de dados através de experimentos em forno resistivo de laboratório; ii) estudo termodinâmico computacional através do software FactSage; iii) comparação e discussão dos resultados obtidos. O objetivo geral será realizar o estudo termodinâmico e experimental a respeito da modificação de inclusões não metálicas através do tratamento com cálcio no aço SAE 1141 . Os objetivos específicos são realizar esse estudo em diferentes temperaturas e concentrações de cálcio no banho, além de diferentes tempos de processo. Como resultado final, espera-se gerar um maior conhecimento que contribua para a compreensão dos fenômenos envolvidos, além de validar o uso do software FactSage para o estudo do perfil inclusionário do aço em questão.
\end{abstract}

Palavras-chave: Inclusões; Tratamento com cálcio.

\section{STUDY OF ADVANCE IN NON-METALLIC INCLUSIONS THROUGH CALCIUM TREATMENT OF SAE 1141 STEEL}

\footnotetext{
Abstract

Non-metallic inclusions impurities are present, in a lesser or greater degree, in steels according to the size, morphology and chemical composition from them. If not moderated, this situation corrupts the mechanical and metallurgical properties of the steel. The modification of inclusions with calcium-based alloys is a strong tool used for the production of clean steels. Such processing is strongly influenced by the chemical composition and temperature of the steel. This study will use as methodology: i) obtaining data through experiments in lab resistive furnace; ii) using computational thermodynamic study through the software FactSage; iii) comparison and discussion of the obtained results. The general aim will perform the thermodynamic study and experimental about the modification of nonmetallic inclusions by treatment with calcium in the SAE 1141 steel. The specific objectives are to conduct this study at different temperatures and concentrations of calcium in the bath, and in a different time process. The end result is expected to generate a greater knowledge that contributes.

Keywords: Inclusions; Calcium treatment.

1 Contribuição técnica ao $68^{\circ}$ Congresso Anual da ABM - Internacional, 30 de julho a 2 de agosto de 2013, Belo Horizonte, MG, Brasil.

2 Graduanda em Engenharia Metalúrgica. Laboratório de Siderurgia, UFRGS, Porto Alegre, RS, Brasil.

3 Engenheiro Metalúrgico. Dr. Professor, Laboratório de Siderurgia, LASID; Depto. de Metalurgia, UFRGS, Porto Alegre, RS, Brasil. wagner@ct.ufrgs.br

4 Engenheiro Metalúrgico. Dr.-Ing. Professor, Laboratório de Siderurgia, LASID; Depto. de Metalurgia, UFRGS, Porto Alegre, RS, Brasil.
} 


\section{INTRODUÇÃO}

Aços de alta usinabilidade que possuem teor elevado de enxofre, são comumente semi-acalmados e seu teor final de oxigênio não é satisfatório para uso em componentes de alto desempenho.

Assim, o alumínio é uma opção largamente utilizada para reduzir o teor de oxigênio desse material, mesmo que seu uso como desoxidante resulte na formação de inclusões não-metálicas sólidas que, por sua vez, podem causar obstrução de válvulas no lingotamento contínuo.

A forma dos cavacos é determinante na velocidade de usinagem. Metais, ao serem usinados, devem produzir um cavaco frágil e, portanto, quebradiço, o que propicia uma maior facilidade de manuseio e operação. ${ }^{(1)}$ A adição de enxofre ao aço é muito conhecida e utilizada a fim de conduzir a formação de sulfetos. Tais inclusões possuem baixo ponto de fusão e alta deformabilidade. O efeito é positivo na usinabilidade, ao agirem como lubrificantes no corte, facilitando a quebra do cavaco na zona de cisalhamento. Assim como no emprego do Alumínio no refino, o emprego do enxofre como formador de sulfetos é agente causador de inclusões nãometálicas.

O potencial do tratamento com cálcio em aços desoxidados ao alumínio é reconhecido e a modificação de inclusões com ligas à base de cálcio é uma ferramenta fortemente utilizada para a produção de aços mais limpos. As características termodinâmicas e cinéticas do tratamento de inclusões não-metálicas com cálcio são fortemente influenciadas pela temperatura e composição química do aço, porém ainda não são completamente conhecidas

Este trabalho aborda um estudo termodinâmico e experimental a respeito da modificação de inclusões não metálicas através do tratamento com cálcio no aço SAE 1141, objetivando especificamente i) obter perfil inclusionário do aço após o tratamento em questão; ii) realizar a comparação de dados obtidos via simulação termodinâmica com dados experimentais e; 3) testar nova configuração de temperaturas e composição para esse modelo de estudo.

\section{MATERIAIS E MÉTODOS}

O aço estudado é o SAE 1141, classificado pela SAE-AISI como um aço carbono ressulfurado. É um material com boa usinabilidade, temperabilidade, conformabilidade e baixa soldabilidade. A metodologia é baseada em estudo realizado anteriormente por Bielefeldt. ${ }^{(2)} \mathrm{Na}$ Tabela 1 , verifica-se a faixa de concentração dos principais elementos de liga do aço em questão.

Tabela 1 - Composição química para o aço SAE 1141 em \% em massa. ${ }^{(3)}$

\begin{tabular}{|c|c|c|c|}
\hline $\mathrm{C}$ & $\mathrm{Mn}$ & $\mathrm{S}$ & $\mathrm{P}$ \\
\hline $0,37-0,45$ & $1,35-1,65$ & $0,08-0,13$ & 0,040 máx. \\
\hline
\end{tabular}

\subsection{Ensaios em Laboratório}

Foram realizados cinco ensaios, sendo quatro deles analisados no presente trabalho, ensaios B1, B2, B3 e B5, de composição semelhante, em forno elétrico resistivo. Os experimentos ocorreram sob atmosfera de argônio, inerte, e a uma taxa de aquecimento de $5^{\circ} \mathrm{C} / \mathrm{min}$. 
A carga é constituída da mistura de ferro de alta pureza com as ligas usadas industrialmente na produção do aço SAE 1141 (Tabela 2). A carga total de cada ensaio tem como massa $1 \mathrm{~kg}$ e as fusões foram realizadas em cadinhos de alumina pura.

Tabela 2 - Ligas utilizadas nos experimentos para $1 \mathrm{~kg}$ de carga, em gramas

\begin{tabular}{|c|c|c|c|c|c|c|}
\hline Mn eletr & Grafita & Enxofre & $\mathrm{Fe}_{2} \mathrm{O}_{3}$ & $\mathrm{FeCrBC}$ & Nieletr & Si puro \\
\hline 15,5 & 5,0 & 1,01 & 0,075 & 2,45 & 0,44 & 1,1 \\
\hline
\end{tabular}

Testaram-se duas disposições de ensaio, chamadas de T1 e T2 (Figuras 1 e 2):

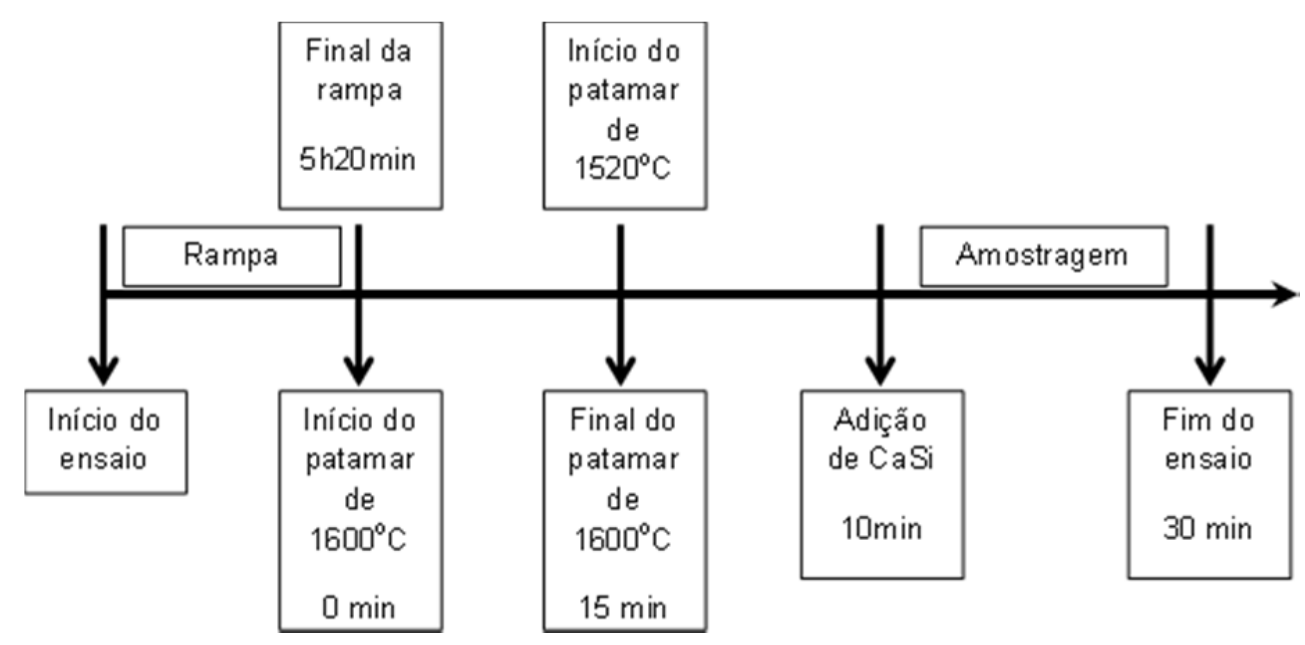

Figura 1 - Fluxo do processo para a condição experimental T1.

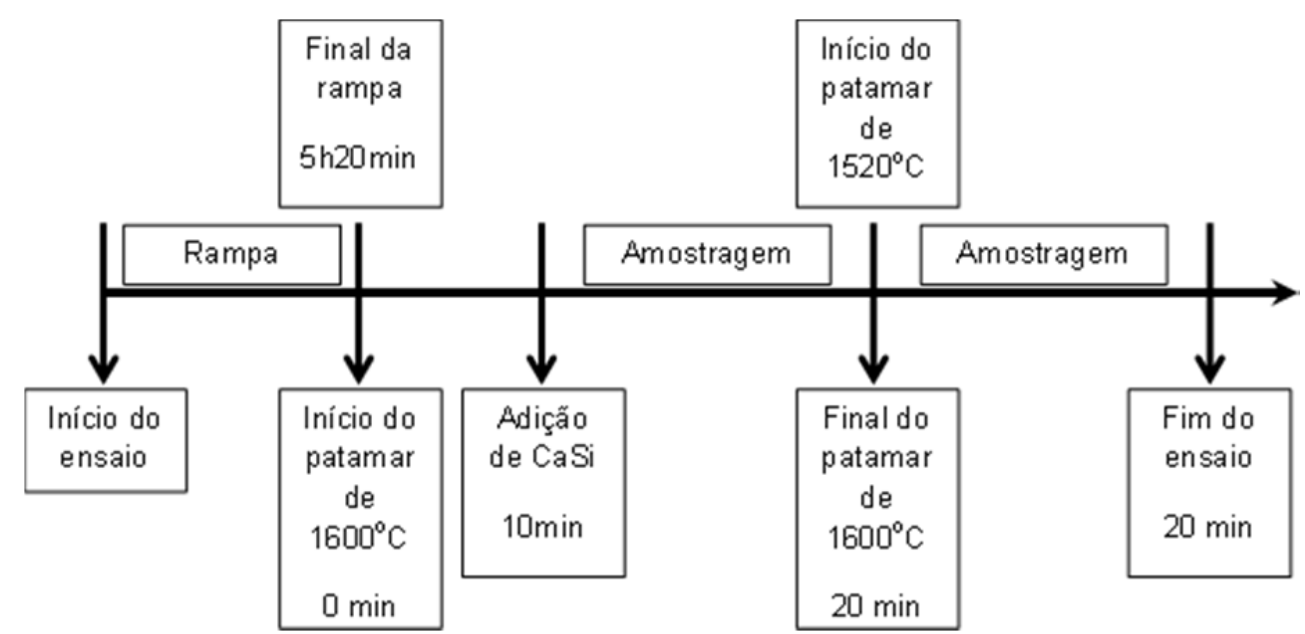

Figura 2 - Fluxo do processo para a condição experimental T2.

O objetivo principal foi o de tentar maximizar o rendimento de CaSi, ou seja, incorporar uma maior quantidade de cálcio ao aço.

$\mathrm{Na}$ amostragem da condição T1 foram coletadas 4 amostras em intervalos de 2 min cada. $\mathrm{Na}$ amostragem da condição T2 foram coletadas duas amostras em cada temperatura, em intervalos de 2 min cada. 
A tabela 3 mostra a relação de ensaios realizados e as condições de cada um.

Tabela 3 - Relação de ensaios realizados

\begin{tabular}{|c|c|c|c|}
\hline & $\begin{array}{c}\text { Condição } \\
\text { experimental }\end{array}$ & $\begin{array}{c}\text { Massa de CaSi } \\
\text { adicionada (g) }\end{array}$ & $\begin{array}{c}\text { Temperatura de } \\
\text { adição de CaSi }\end{array}$ \\
\hline B1 & T1 & 0,95 & $1.520^{\circ} \mathrm{C}$ \\
\hline B2 & T1 & 0,95 & $1.520^{\circ} \mathrm{C}$ \\
\hline B3 & T2 & 0,95 & $1.600^{\circ} \mathrm{C}$ \\
\hline B5 & T2 & 0,68 & $1.520^{\circ} \mathrm{C}$ \\
\hline
\end{tabular}

\subsection{Preparação e Análise das Amostras}

As amostras coletadas foram cortadas em pinos de aproximadamente $1,5 \mathrm{~cm}$, embutidas e submetidas à preparação metalográfica convencional. Posteriormente, foram analisadas em Microscópio Eletrônico de Varredura (MEV/EDS) marca JEOL modelo 5800, no Centro de Microscopia Eletrônica da UFRGS.

Foram analisados quatro pinos dos ensaios B1 e B5 e três do ensaio B2 e B3 porque houve problema de preenchimento nas demais amostras, impedindo as análises.

É relevante relatar que, após algumas tentativas, os autores concluíram ser vantajoso, para análise das amostras em questão, o uso do MEV em Elétrons Retroespalhados (backscatteringelectron) e tensão de 20kv. É importante, para obter uma análise satisfatória, que haja um equilíbrio da tensão e dos elétrons emitidos utilizados para obtenção da imagem.

A imagem gerada por elétrons retroespalhados fornece diferentes informações em relação ao contraste que apresentam: além de uma imagem topográfica também se obtém uma imagem em função do número atômico dos elementos presentes na amostra. A amplificação é independente do foco da lente e pode se variar rapidamente centralizando-se num ponto fixo da superfície. Embora usando exposição a duas dimensões, as imagens produzidas são caracterizadas por seu aspecto tridimensional e por níveis de cinza relacionados à composição da superfície da amostra. As imagens produzidas pelo detector de BSE (elétrons retroespalhados) são imagens de relativa facilidade de interpretação por equipe não especializada. Os elétrons retroespalhados possuem uma energia que é em torno daquela do feixe incidente (choques elásticos). A resolução será, portanto, maior à medida que a tensão for mais fraca. Mas é preciso salientar o fato de que se produzem menos elétrons retroespalhados à medida que a tensão diminui. ${ }^{(4)}$

\subsection{Amostras para Análise Química}

O aço remanescente no cadinho foi analisado em Espectrômetro de Emissão Ótica, sendo analisados seis pontos por ensaio a fim de se verificar se a composição ensaiada estava de acordo com o especificado para o aço SAE 1141, bem como para análise de oxigênio total, realizada em duas amostras por ensaio, no Determinador Simultâneo de Nitrogênio e Oxigênio, marca LECO modelo 436. 


\subsection{Simulações Termodinâmica}

A simulação termodinâmica computacional foi realizada através do software comercial FactSage, versão 6.3. Utilizou-se dois bancos de dados, um para aço (FSstel-LIQU) e o outro para óxidos (FToxid-SLAGA). Foi utilizado o móduloEquilib, módulo de minimização da energia livre de Gibbs.

Os dados de entrada foram: os resultados das análises das amostras de aço feitas no Espectrômetro de Emissão ótica e Determinador Simultâneo de Nitrogênio e Oxigênio e as temperaturasde $1600^{\circ} \mathrm{C}$ (para representar a condição em uma panela siderúrgica) e $1520^{\circ} \mathrm{C}$ (para representar as condições em um distribuidor de lingotamento contínuo).

\section{RESULTADOS E DISCUSSÃO}

\subsection{Composição Química}

Nas Tabelas 4 a 7 encontram-se os resultados da análise química do aço ensaiado remanescente no cadinho. Os teores de $\mathrm{Al}, \mathrm{Ca}, \mathrm{O}_{\text {total }}$ e $\mathrm{N}$ estão em ppm. A presença de magnésio não foi analisada, mas estimada em 1ppm, como dado para as simulações termodinâmicas.

Tabela 4- Composição química média do aço do ensaio B1 em \% em massa

\begin{tabular}{|c|c|c|c|c|c|c|c|c|c|c|c|c|}
\hline B1 & $\mathrm{C}$ & $\mathrm{Si}$ & $\mathrm{Mn}$ & $\mathrm{P}$ & $\mathrm{S}$ & $\mathrm{Cr}$ & $\mathrm{Ni}$ & $\mathrm{Mo}$ & $\mathrm{Al}$ & $\mathrm{Ca}^{*}$ & $\mathrm{O}_{\text {total }}$ & $\mathrm{N}^{*}$ \\
\hline Média & 0,38 & 0,18 & 1,53 & 0,01 & 0,11 & 0,20 & 0,07 & 0,01 & 20,00 & 10,00 & 27,50 & 4,50 \\
\hline Desvio & 0,00 & 0,00 & 0,01 & 0,00 & 0,01 & 0,01 & 0,00 & 0,00 & 0,00 & 2,61 & 7,78 & 0,70 \\
\hline
\end{tabular}

Tabela 5- Composição química média do aço do ensaio B2 em \% em massa

\begin{tabular}{|c|c|c|c|c|c|c|c|c|c|c|c|c|}
\hline B2 & $\mathrm{C}$ & $\mathrm{Si}$ & $\mathrm{Mn}$ & $\mathrm{P}$ & $\mathrm{S}$ & $\mathrm{Cr}$ & $\mathrm{Ni}$ & $\mathrm{Mo}$ & $\mathrm{Al}^{*}$ & $\mathrm{Ca}^{*}$ & $\mathrm{O}_{\text {total }}$ & $\mathrm{N}^{*}$ \\
\hline Média & 0,37 & 0,21 & 1,39 & 0,02 & 0,09 & 0,19 & 0,07 & 0,01 & 41,67 & 13,83 & 33,50 & 4,00 \\
\hline Desvio & 0,00 & 0,00 & 0,08 & 0,00 & 0,01 & 0,00 & 0,00 & 0,00 & 4,08 & 1,72 & 2,12 & 0,00 \\
\hline
\end{tabular}

*em ppm

Tabela 6-Composição química média do aço do ensaio B3 em \% em massa

\begin{tabular}{|c|c|c|c|c|c|c|c|c|c|c|c|c|}
\hline B3 & $\mathrm{C}$ & $\mathrm{Si}$ & $\mathrm{Mn}$ & $\mathrm{P}$ & $\mathrm{S}$ & $\mathrm{Cr}$ & $\mathrm{Ni}$ & $\mathrm{Mo}$ & $\mathrm{Al}^{\star}$ & $\mathrm{Ca}^{*}$ & $\mathrm{O}_{\text {total }}$ & $\mathrm{N}^{*}$ \\
\hline Média & 0,38 & 0,21 & 1,56 & 0,01 & 0,09 & 0,19 & 0,07 & 0,01 & 46,70 & 19,80 & 42,00 & 4,00 \\
\hline Desvio & 0,01 & 0,00 & 0,06 & 0,00 & 0,02 & 0,00 & 0,00 & 0,00 & 5,16 & 3,27 & 2,00 & 0,00 \\
\hline
\end{tabular}

Tabela 7- Composição química média do aço do ensaio B5 em \% em massa

\begin{tabular}{|c|c|c|c|c|c|c|c|c|c|c|c|c|}
\hline B5 & $\mathrm{C}$ & $\mathrm{Si}$ & $\mathrm{Mn}$ & $\mathrm{P}$ & $\mathrm{S}$ & $\mathrm{Cr}$ & $\mathrm{Ni}$ & $\mathrm{Mo}$ & $\mathrm{Al}^{\star}$ & $\mathrm{Ca}^{*}$ & $\mathrm{O}_{\text {total }}$ & $\mathrm{N}^{*}$ \\
\hline Média & 0,40 & 0,18 & 1,50 & 0,01 & 0,10 & 0,20 & 0,07 & 0,01 & 32,00 & 16,75 & 39,00 & 6,00 \\
\hline Desvio & 0,00 & 0,00 & 0,02 & 0,00 & 0,03 & 0,00 & 0,00 & 0,00 & 4,47 & 1,26 & 1,41 & 0,00 \\
\hline
\end{tabular}

Comparando os ensaios B1, B2 e B3 - mesma quantidade de CaSi adicionado - pode-se observar que, no ensaio B3 foi obtido o maior teor de Ca no aço. Isso devido a maior temperatura do ensaio B3 no memento da adição de CaSi.

Comparando os ensaios B3 e B5 - mesma temperatura de adição de CaSi - o ensaio B3 teve um maior rendimento de $\mathrm{Ca}$, porém com desvio padrão entre os pontos analisados mais elevado. Isso mostra que a homogeneização de cálcio tende a ser maior em experimento com menor quantidade de CaSi adicionada. 
Os elementos Ca e Al são importantes na formação de inclusões. Dessa forma, a homogeneidade das amostras é tema de constante análise. Na Figura 3, é mostrada a "fatia" de aço e a distribuição dos pontos onde é feita a análise química.

A Tabela 8 mostra o teor de Al e Ca em cada ponto dos aços ensaiados. Observa-se que os pontos 3 e 4, de localização central onde a liga é injetada, possuem teor alto de cálcio. Há duas hipóteses possíveis: a dissolução não uniforme da liga de CaSi e a presença de porosidades no centro da amostra, o que pode gerar resultados incorretos de análise. Uma sugestão para trabalhos futuros será analisar também os pinos retirados durante os ensaios.

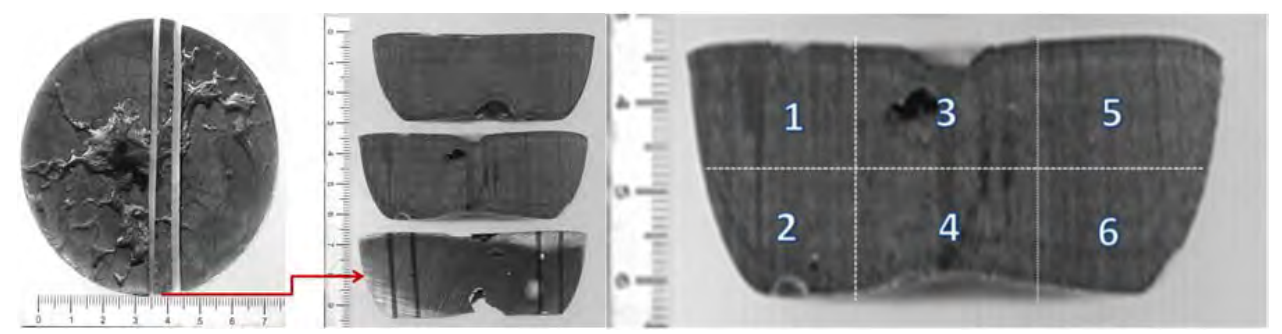

Figura 3 - Vista superior da bolacha de aço e o produto do corte transversal.

Tabela 8-Teor de Al e Ca dos aços ensaiados em ppm

\begin{tabular}{|c|c|c|c|c|c|c|c|c|}
\hline & \multicolumn{2}{|c|}{ B1 } & \multicolumn{2}{c|}{ B2 } & \multicolumn{2}{c|}{ B3 } & \multicolumn{2}{c|}{ B5 } \\
\hline Ponto & Al & Ca & Al & Ca & Al & Ca & Al & Ca \\
\hline $\mathbf{1}$ & 20 & 7 & 40 & 12 & 50 & 18 & 30 & 15 \\
\hline $\mathbf{2}$ & 20 & 8 & 40 & 16 & 40 & 15 & 30 & 17 \\
\hline $\mathbf{3}$ & 20 & 14 & 40 & 15 & 50 & 23 & 30 & 18 \\
\hline $\mathbf{4}$ & 20 & 12 & 40 & 12 & 40 & 37 & 60 & 46 \\
\hline $\mathbf{5}$ & 20 & 9 & 40 & 13 & 50 & 22 & 30 & 17 \\
\hline $\mathbf{6}$ & 20 & 10 & 50 & 15 & 50 & 21 & 40 & 36 \\
\hline
\end{tabular}

\subsection{Composição das Inclusões x Simulação Termodinâmica}

Nos ensaios $\mathrm{B} 1$ e B2, foram observados dois grupos de inclusões. O primeiro no sistema $\mathrm{MnO}-\mathrm{Al}_{2} \mathrm{O}_{3}-\mathrm{SiO}_{2}$ com traços de $\mathrm{MgO}$ e $\mathrm{CaO}$. O segundo grupo de inclusões apresentou um alto percentual em massa de $\mathrm{CaO}$, maior do que $80 \%$. Isso mostra que, nos dois ensaios, não ocorreu a homogeneização do banho de forma satisfatória. O primeiro grupo de inclusões representa a desoxidação com $\mathrm{Mn}$ e Si, com Al residual. O segundo grupo mostra que a liga de CaSi não se difundiu de forma adequada. A presença de traços de $\mathrm{MgO}$ se deve a presença de $\mathrm{Mg}$ residual nas ligas utilizadas.

A Tabela 9 mostra o resultado de média e desvio da análise de inclusões de todas as amostras dos experimentos B3 e B5. As inclusões estão no sistema (CaO, $\mathrm{MnO})-\mathrm{Al}_{2} \mathrm{O}_{3}-\mathrm{SiO}_{2}$. O $\mathrm{CaO}$ tende a substituir o $\mathrm{MnO}$ no produto de desoxidação a medida que o cálcio interage com as inclusões formadas. A proporção de CaO e $\mathrm{MnO}$ depende do grau de oxidação do banho. Menores teores de oxigênio total implicam em maiores teores de $\mathrm{CaO}$ nas inclusões. O oxigênio total da $\mathrm{B} 5$ foi ligeiramente menor do que na $\mathrm{B} 3$. $\mathrm{O}$ teor de $\mathrm{Al}_{2} \mathrm{O}_{3}$ nas inclusões da $\mathrm{B} 3$ foi maior do que na B5 devido ao maior teor de Al no aço (Tabelas 6 e 7). A presença de enxofre pode estar associada a CaS e/ou MnS nas inclusões. Observa-se também a presença de $\mathrm{MgO}$ nas inclusões. Magnésio pode estar presente no alumínio adicionado, por exemplo. 
Tabela 9-Resultado da análise de inclusões via MEV/EDS das amostras B3 e B5 Amostra B3 - Geral (20 inclusões)

\begin{tabular}{c|c|c|c|c|c|c|} 
& $\mathrm{MgO}$ & $\mathrm{Al}_{2} \mathrm{O}_{3}$ & $\mathrm{SiO}_{2}$ & $\mathrm{~S}$ & $\mathrm{CaO}$ & $\mathrm{MnO}$ \\
\cline { 2 - 7 } Média & 5,10 & 32,04 & 17,66 & 2,70 & 31,27 & 11,24 \\
\cline { 2 - 7 } Desvio & 4,47 & 12,34 & 7,57 & 1,94 & 7,60 & 6,92 \\
\hline
\end{tabular}

Amostra B5 - Geral (32 inclusões)

\begin{tabular}{l|c|c|c|c|c|c|} 
& $\mathrm{MgO}$ & $\mathrm{Al}_{2} \mathrm{O}_{3}$ & $\mathrm{SiO}_{2}$ & $\mathrm{~S}$ & $\mathrm{CaO}$ & $\mathrm{MnO}$ \\
\cline { 2 - 7 } Média & 1,09 & 24,78 & 29,79 & 2,39 & 34,72 & 7,23 \\
\cline { 2 - 7 } Desvio & 0,93 & 8,17 & 3,50 & 1,68 & 6,91 & 3,07 \\
\hline
\end{tabular}

A Tabela 10 mostra o resultado da análise das inclusões obtida nos pinos retirados ao longo do tempo de ensaio (média e desvio). Na amostra B3-1, podemse observar dois grupos de inclusões. O primeiro grupo de inclusões foi modificado pelo cálcio, $\mathrm{CaO}$ predomina sobre $\mathrm{MnO}$. O segundo grupo representa inclusões remanescentes da desoxidação, com menor teor de CaO incorporado. As amostras B3-3 e B3-4 foram retiradas em um maior tempo após a adição de CaSi, sendo que a amostra B3-4 é mais heterogênea do que a amostras B3-3. As diferenças de composição entre (B3-1) e (B3-3; B3-4) podem ser devido às diferentes temperaturas $\left(1600^{\circ} \mathrm{C}\right.$ para $\left.\mathrm{B} 3-1\right)$ e $\left(1520^{\circ} \mathrm{C}\right.$ para $\mathrm{B} 3-3$; $\left.\mathrm{B} 3-4\right)$ e tempo transcorrido após a adição de CaSi (B3-1 foi a primeira amostra retirada).

Tabela 10-Resultado da análise de inclusões via MEV/EDS das amostras do ensaio B3

Amostra B3 - 1

\begin{tabular}{c|c|c|c|c|c|c|c|}
\cline { 2 - 7 } Grupo 1 & $\mathrm{MgO}$ & $\mathrm{Al}_{2} \mathrm{O}_{3}$ & $\mathrm{SiO}_{2}$ & $\mathrm{~S}$ & $\mathrm{CaO}$ & $\mathrm{MnO}$ \\
\cline { 2 - 8 } & Média & 6,69 & 29,88 & 16,93 & 2,25 & 34,04 & 10,22 \\
\cline { 2 - 8 } & Desvio & 7,47 & 21,75 & 3,13 & 1,96 & 11,62 & 6,39 \\
\hline \multirow{7}{*}{ Grupo 2} & Média & 2,08 & 5,91 & 30,88 & 2,21 & 12,20 & 46,72 \\
\cline { 2 - 8 } & Desvio & 1,54 & 1,85 & 11,37 & 2,45 & 5,15 & 14,62 \\
\hline
\end{tabular}

Amostra B3 - 3

\begin{tabular}{|c|c|c|c|c|c|c|}
\cline { 2 - 7 } \multicolumn{1}{c|}{} & $\mathrm{MgO}$ & $\mathrm{Al}_{2} \mathrm{O}_{3}$ & $\mathrm{SiO}_{2}$ & $\mathrm{~S}$ & $\mathrm{CaO}$ & $\mathrm{MnO}$ \\
\hline Média & 4,77 & 36,19 & 18,05 & 2,33 & 31,40 & 7,26 \\
\hline Desvio & 1,98 & 3,81 & 2,10 & 1,22 & 3,77 & 3,33 \\
\hline
\end{tabular}

Amostra B3 - 4

\begin{tabular}{|c|c|c|c|c|c|c|}
\cline { 2 - 7 } \multicolumn{1}{c|}{} & $\mathrm{MgO}$ & $\mathrm{Al}_{2} \mathrm{O}_{3}$ & $\mathrm{SiO}_{2}$ & $\mathrm{~S}$ & $\mathrm{CaO}$ & $\mathrm{MnO}$ \\
\hline Média & 4,54 & 24,63 & 17,38 & 3,89 & 28,75 & 20,81 \\
\hline Desvio & 6,35 & 14,54 & 15,90 & 2,98 & 11,03 & 3,14 \\
\hline
\end{tabular}

A Tabela 11 mostra os resultados de média e desvio para as amostras coletadas ao longo do tempo no ensaio B5. A modificação das inclusões pelo cálcio é rápida, o que está de acordo com a literatura, ainda que os mecanismos não sejam plenamente conhecidos. O que chama mais atenção é a variação do teor de $\mathrm{Al}_{2} \mathrm{O}_{3}$ da amostra B5-1 em relação às demais amostras. Talvez tenha ocorrido uma homogeneização mais lenta do Al. 
Tabela 11-Resultado da análise de inclusões via MEV/EDS das amostras do ensaio B5 Amostra B5 -1

\begin{tabular}{|c|c|c|c|c|c|c|}
\cline { 2 - 7 } \multicolumn{1}{c|}{} & $\mathrm{MgO}$ & $\mathrm{Al}_{2} \mathrm{O}_{3}$ & $\mathrm{SiO}_{2}$ & $\mathrm{~S}$ & $\mathrm{CaO}$ & $\mathrm{MnO}$ \\
\hline Média & 0,50 & 14,06 & 33,30 & 3,76 & 40,33 & 8,06 \\
\hline Desvio & 0,85 & 4,11 & 1,30 & 1,20 & 5,11 & 2,66 \\
\hline
\end{tabular}

Amostra B5 - 3

\begin{tabular}{|c|c|c|c|c|c|c|}
\cline { 2 - 7 } \multicolumn{1}{c|}{} & $\mathrm{MgO}$ & $\mathrm{Al}_{2} \mathrm{O}_{3}$ & $\mathrm{SiO}_{2}$ & $\mathrm{~S}$ & $\mathrm{CaO}$ & $\mathrm{MnO}$ \\
\hline Média & 1,68 & 28,97 & 29,02 & 2,26 & 30,27 & 7,80 \\
\hline Desvio & 0,88 & 2,29 & 3,24 & 1,89 & 4,27 & 3,84 \\
\hline
\end{tabular}

Amostra B5 - 4

\begin{tabular}{|c|c|c|c|c|c|c|}
\cline { 2 - 7 } \multicolumn{1}{c|}{} & $\mathrm{MgO}$ & $\mathrm{Al}_{2} \mathrm{O}_{3}$ & $\mathrm{SiO}_{2}$ & $\mathrm{~S}$ & $\mathrm{CaO}$ & $\mathrm{MnO}$ \\
\hline Média & 0,98 & 30,46 & 27,20 & 1,16 & 34,46 & 5,73 \\
\hline Desvio & 0,68 & 4,46 & 2,39 & 0,40 & 7,40 & 1,93 \\
\hline
\end{tabular}

As Tabelas 12 e 13 mostram uma comparação entre os resultados obtidos via MEV/EDS com as simulações via FactSage. No geral existe uma boa correlação entre os resultados, mostrando que as inclusões estavam próximas do estado de equilíbrio termodinâmico. Foram realizadas simulações para as temperaturas de $1.520^{\circ} \mathrm{C}$ e $1.600^{\circ} \mathrm{C}$. Na temperatura de $1520^{\circ} \mathrm{C}$, ocorre maior formação de $\mathrm{Al}_{2} \mathrm{O}_{3} \mathrm{com}$ queda do teor de $\mathrm{CaO}$ nas inclusões, o que é esperado.

Tabela 12-Comparação dos resultados da análise de inclusões via MEV/EDS e simulação via FactSage do ensaio B3

Amostra B3 - Geral (20 inclusões)

\begin{tabular}{|c|c|c|c|c|c|c|}
\cline { 2 - 7 } \multicolumn{1}{c|}{} & $\mathrm{MgO}$ & $\mathrm{Al}_{2} \mathrm{O}_{3}$ & $\mathrm{SiO}_{2}$ & $\mathrm{~S}$ & $\mathrm{CaO}$ & $\mathrm{MnO}$ \\
\hline Média & 5,10 & 32,04 & 17,66 & 2,70 & 31,27 & 11,24 \\
\hline Desvio & 4,47 & 12,34 & 7,57 & 1,94 & 7,60 & 6,92 \\
\hline
\end{tabular}

Amostra B3 - Simulação a $\mathrm{T}=1600^{\circ} \mathrm{C}$

\begin{tabular}{|c|c|c|c|c|c|}
\hline $\mathrm{MgO}$ & $\mathrm{Al}_{2} \mathrm{O}_{3}$ & $\mathrm{SiO}_{2}$ & $\mathrm{~S}$ & $\mathrm{CaO}$ & $\mathrm{MnO}$ \\
\hline 1,64 & 32,13 & 25,47 & 0,14 & 36,29 & 3,80 \\
\hline
\end{tabular}

Amostra B3 - Simulação a $\mathrm{T}=1520^{\circ} \mathrm{C}$

\begin{tabular}{|c|c|c|c|c|c|}
\hline $\mathrm{MgO}$ & $\mathrm{Al}_{2} \mathrm{O}_{3}$ & $\mathrm{SiO}_{2}$ & $\mathrm{~S}$ & $\mathrm{CaO}$ & $\mathrm{MnO}$ \\
\hline 1,73 & 40,54 & 21,95 & 0,12 & 31,45 & 3,77 \\
\hline
\end{tabular}

Tabela 13-Comparação dos resultados da análise de inclusões via MEV/EDS e simulação via FactSage do ensaio B5

Amostra B5 - Geral (32 inclusões)

\begin{tabular}{l|c|c|c|c|c|c|} 
& $\mathrm{MgO}$ & $\mathrm{Al}_{2} \mathrm{O}_{3}$ & $\mathrm{SiO}_{2}$ & $\mathrm{~S}$ & $\mathrm{CaO}$ & $\mathrm{MnO}$ \\
\cline { 2 - 7 } Média & 1,09 & 24,78 & 29,79 & 2,39 & 34,72 & 7,23 \\
\cline { 2 - 7 } Desvio & 0,93 & 8,17 & 3,50 & 1,68 & 6,91 & 3,07 \\
\hline
\end{tabular}

Amostra B5 - Simulação a $\mathrm{T}=1600^{\circ} \mathrm{C}$

\begin{tabular}{|c|c|c|c|c|c|}
\hline $\mathrm{MgO}$ & $\mathrm{Al}_{2} \mathrm{O}_{3}$ & $\mathrm{SiO}_{2}$ & $\mathrm{~S}$ & $\mathrm{CaO}$ & $\mathrm{MnO}$ \\
\hline 1,93 & 27,97 & 28,11 & 0,12 & 36,76 & 4,56 \\
\hline
\end{tabular}

Amostra B5 - Simulação a $\mathrm{T}=1520^{\circ} \mathrm{C}$

\begin{tabular}{|c|c|c|c|c|c|}
\hline $\mathrm{MgO}$ & $\mathrm{Al}_{2} \mathrm{O}_{3}$ & $\mathrm{SiO}_{2}$ & $\mathrm{~S}$ & $\mathrm{CaO}$ & $\mathrm{MnO}$ \\
\hline 2,00 & 35,48 & 25,76 & 0,09 & 30,49 & 5,71 \\
\hline
\end{tabular}


A Figura 4 mostra a média da análise de inclusões via MEV/EDS inseridas no diagrama ternário $\mathrm{CaO}-\mathrm{SiO}_{2}-\mathrm{Al}_{2} \mathrm{O}_{3}{ }^{(5)}$ Os resultados foram normalizados para um sistema de três componentes. Os dois ensaios estão na área totalmente líquida a $1600^{\circ} \mathrm{C}$. Na temperatura de $1.520^{\circ} \mathrm{C}$, pode haver alguma precipitação de fase sólida $\left(\mathrm{C}_{2} \mathrm{AS}\right)$, que não apresenta características de obstrução de válvulas no lingotamento contínuo.

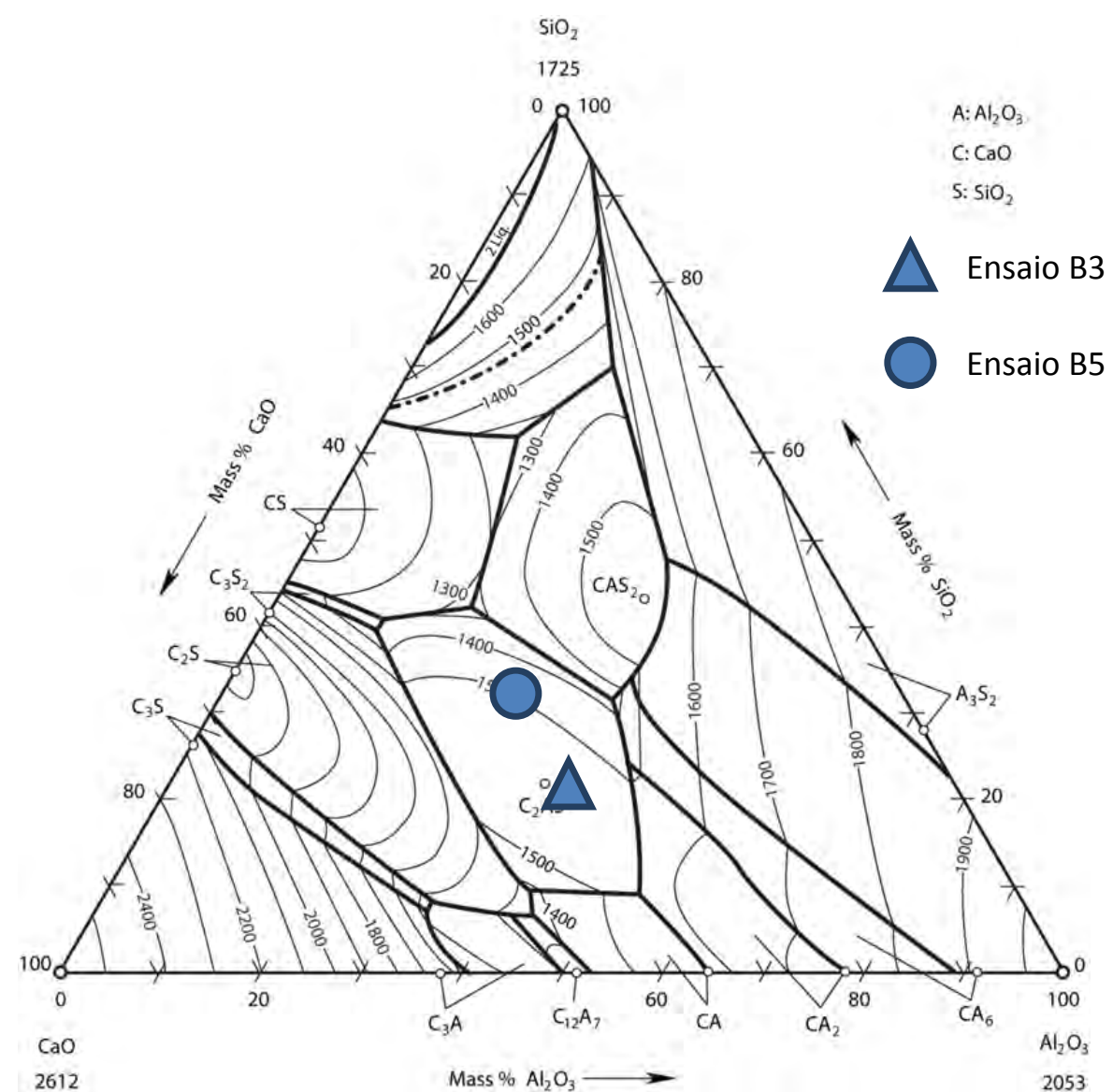

Figura 4 - Diagrama ternário $\mathrm{CaO}-\mathrm{SiO}_{2}-\mathrm{Al}_{2} \mathrm{O}_{3}$ e os resultados dos ensaios $\mathrm{B} 3$ e $\mathrm{B} 5 .{ }^{(5)}$

É de extrema relevância relatar que as inclusões encontradas nos ensaios B3 e B5 são em suma globulares, conforme exemplos na Figura 5. Tal morfologia indica que possivelmente as inclusões no banho eram líquidas e, então, não provocariam entupimento de válvulas.

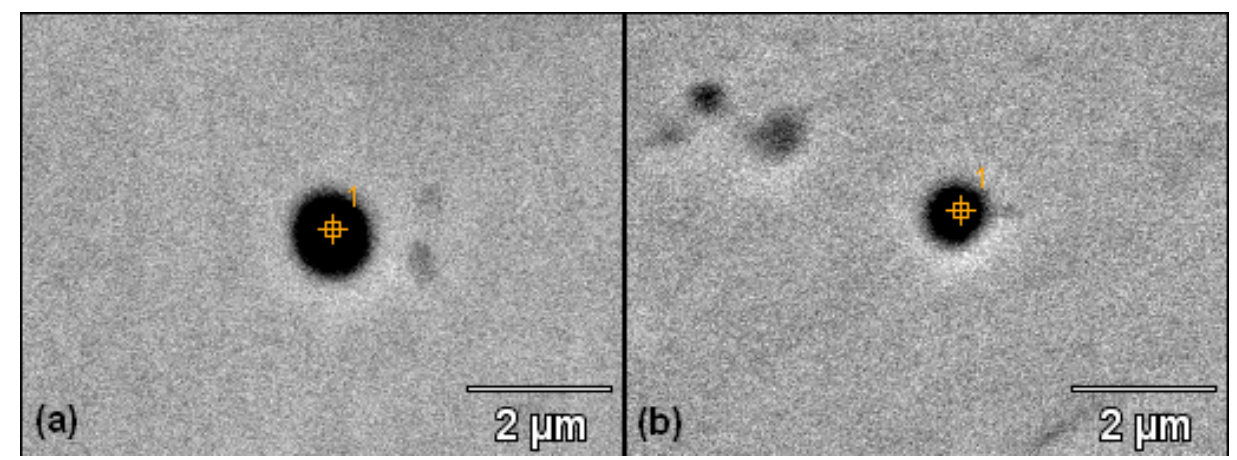

Figura 5 - Inclusões analisadas por MEV/EDS. a) ensaio B3. b) ensaio B5. 
Também foram analisadas via MEV/EDS amostras do aço remanescente nos ensaios B1 e B5, em ambas as análises foram observadas inclusões ricas em sulfeto de manganês (MnS) (Figura 6). Era esperada a presença dessas inclusões no aço remanescente e não nos pinos coletados porque o enxofre é solúvel no aço fundido, mas no aço sólido sua solubilização é muito lenta. Ele é precipitado sob a forma de sulfetos de metal durante a solidificação e o padrão de precipitação é influenciado pela segregação. ${ }^{(6)}$

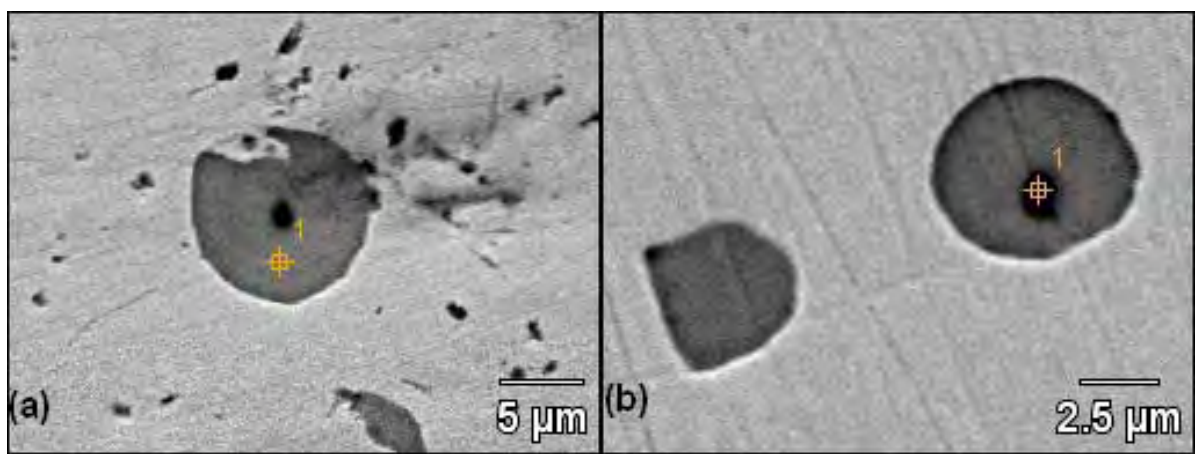

Figura 6 - Inclusões de Sulfeto de Manganês analisadas por MEV/EDS. A) ensaio B1. b) ensaio B5.

\section{CONCLUSÕES}

Neste estudo foi avaliada a modificação das inclusões com o tratamento com cálcio para o aço SAE 1141, em escala laboratorial. O trabalho foi eficaz em reproduzir o aço em questão.

Parte das inclusões nos ensaios B1 e B2 são extremamente ricas em CaO devido a não homogeneização da liga no banho evidenciando possível ineficiência da disposição de temperatura T1 em experimentos com adição tão elevada de cálcio. Esses resultados mostram a necessidade de se obter um aço homogêneo.

Os resultados dos ensaios B3 e B5, realizados na disposição de temperatura T2, se mostraram mais satisfatórios que os dois anteriores no que se refere a homogeneidade das amostras, harmonia com os dados apurados através da simulação termodinâmica e a morfologia globular das inclusões.

Ainda no ensaio B5, houve maior correlação entre os resultados obtidos via MEV/EDS e a simulação termodinâmica. Tal constatação salienta a maior facilidade de controle de experimentos com adição inferior de cálcio.

O software FactSage conseguiu prever de modo satisfatório o perfil inclusionário do aço analisado.

\section{Agradecimentos}

Os autores agradecem ao Centro de Microscopia Eletrônica da UFRGS pela disponibilidade do MEV-EDS e aos colegas do LaSid pelo auxílio nos experimentos.

\section{REFERÊNCIAS}

1 BAPTISTA, André Luís de Brito: Aspectos metalúrgicos na avaliação da usinabilidade de aços.Rem: Rev. Esc. Minas, Ouro Preto, v. 55, n. 2,Apr. 2002.

2 BIELEFELDT, Wagner Viana. Tratamento de inclusões não-metálicas com cálcio nos aços SAE 1141 e SAE 8620. Porto Alegre: UFRGS, 2009. Tese (Doutorado) - Programa de Pós-Graduação em Engenharia de Minas, Metalúrgica e de Materiais, Escola de Engenharia, Universidade Federal do Rio Grande do Sul, Porto Alegre, 2009. 
3 ASM. Volume 1, Properties and Selection: Irons, Steels, and High Performance Alloys. ASM Handbook.ASM International, 1993.

4 Dedavid, B. A. et al: Microscopia Eletrônica de Varredura - Aplicações e Preparação de Amostras. EDIPUCRS, 2007.

5 SLAG ATLAS., 2. ed. Düsseldorf: VerlagStahleisen $\mathrm{GmbH}, 1995$.

6 KIESSLING, R. Non Metallic Inclusions in Steels Parts I-IV. London: The Institute of Materials, 1978. 